\title{
Haptic curvature comparison of convex and concave shapes
}

\author{
Bernard J van der Horst, Astrid M L Kappers \\ Department of Physics of Man, Helmholtz Instituut, Universiteit Utrecht, Princetonplein 5, \\ NL 3584 CC Utrecht, The Netherlands; e-mail: b.j.vanderhorst@phys.uu.nl \\ Received 23 February 2007, in revised form 5 December 2007; published online 1 August 2008
}

\begin{abstract}
A sculpture and the mould in which it was formed are typical examples of objects with an identical, but opponent, surface shape: each convex (ie outward pointing) surface part of a sculpture has a concave counterpart in the mould. The question arises whether the object features of opponent shapes can be compared by touch. Therefore, we investigated whether human observers were able to discriminate the curvatures of convex and concave shapes, irrespective of whether the shape was convex or concave. Using a 2AFC procedure, subjects had to compare the curvature of a convex shape to the curvature of a concave shape. In addition, results were also obtained for congruent shapes, when the curvature of either only convex shapes or only concave shapes had to be compared. Psychometric curves were fitted to the data to obtain threshold and bias results. When subjects explored the stimuli with a single index finger, significantly higher thresholds were obtained for the opponent shapes than for the congruent shapes. However, when the stimuli were touched by two index fingers, one finger per surface, we found similar thresholds. Systematic biases were found when the curvature of opponent shapes was compared: the curvature of a more curved convex surface was judged equal to the curvature of a less curved concave surface. We conclude that human observers had the ability to compare the curvature of shapes with an opposite direction, but that their performance decreased when they sensed the opponent surfaces with the same finger. Moreover, they systematically underestimated the curvature of convex shapes compared to the curvature of concave shapes.
\end{abstract}

\section{Introduction}

A sculpture and the mould from which it was produced are typical examples of objects with an identical, but opponent, shape. In contrast to the haptic system, the visual system is not always able to distinguish between identical shapes with an opposite direction (for example, the hollow-face illusion). The special feature of the haptic system is that it has direct access to objects. Obviously, subjects are able to discriminate a convex shape from its concave counterpart by touch. However, it is not yet known whether subjects are able to compare the properties of opponent shapes. Until now, studies in haptic shape perception have concentrated on the ability of human observers to compare the properties of similar objects, oriented in the same direction. Inspired by what is known from these studies, we wondered whether human observers are also able to compare the properties of object surfaces with an opponent shape.

The surface of a sculpture, or any smooth object, can be described locally by a single parameter - the curvature. The stimuli we used in this study were circularly curved cylinder parts, each part having a constant curvature value over the stimulus surface. Convex as well as concave shapes were used. By definition, a convex shape curves outwards, whereas a concave shape curves inwards. A convex surface and a concave surface with identical curvature fit into each other like a sculpture and its mould (see figure 1). In the experiments we performed in this study, a distinction was made between congruent modes and opponent modes. In a congruent mode, either two convex stimuli or two concave stimuli were presented to a subject, whereas in an opponent mode, both a convex shape and a concave shape were presented.

The haptic perception of convex and concave shapes has been the subject of several studies. Experiments in which subjects had to distinguish a curved surface from a flat 


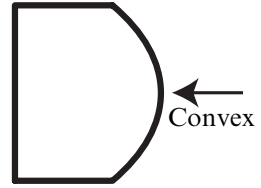

(a)

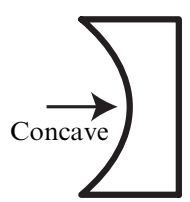

$\angle$

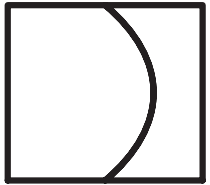

(b)

Figure 1. (a) Illustrations of a convex shape and a concave shape. (b) A convex shape and a concave shape with the same curvatures fit into each other like a sculpture and its mould.

one (detection experiments) revealed no difference in detection threshold for convex and concave shapes, either when spherically curved surfaces were applied to the finger pad (Goodwin et al 1991), or when subjects had to move their finger over zero or second-order Gaussian-shaped surfaces (Louw et al 2000, 2002a). Similar thresholds for convex and concave stimuli were also found in experiments in which subjects had to indicate which of the two stimuli presented felt more curved (discrimination experiments). This was found both in conditions where cylindrically curved, handsized surfaces were actively explored (Kappers and Koenderink 1996), as in conditions where circularly curved strips were felt passively (Pont et al 1997). The curvatures used in these experiments were between $-5.7 \mathrm{~m}^{-1}$ and $+5.7 \mathrm{~m}^{-1}$ and between $-1.8 \mathrm{~m}^{-1}$ and $+1.8 \mathrm{~m}^{-1}$, respectively.

The finding that thresholds were similar for convex and concave shapes might be understood by the fact that the slope difference over the stimulus is a good first approximation for the effective stimulus for the discrimination of curvature (Gordon and Morison 1982; Pont et al 1997, 1999). This concept is supported by a neurophysiological study by LaMotte and Srinivasan (1996). Extensive studies on curved surfaces by Louw et al (2002a, 2002b) confirmed that subjects were particularly sensitive to the slope difference of the stimulus, but that the curvature of the stimulus was also of significant importance. Since a convex shape and its concave counterpart can be characterised by an identical slope difference and an identical curvature, it follows that their discrimination thresholds are the same. In the results reviewed so far, subjects had to compare the properties of convex surfaces to those of other convex surfaces, or the properties of concave surfaces to those of other concave surfaces. Asking subjects to compare the properties of a convex surface to the properties of a concave surface is quite a different matter. Specifically, subjects can be asked which of two surfaces presented feels the more curved. When subjects are able to perform this task by comparing the absolute values of the slope differences and curvatures of the stimuli directly, they will encounter thresholds similar to those they encounter when they have to compare convex (concave) shapes to other convex (concave) shapes. However, if the direction of the surface with respect to the external space or with respect to the finger influences their sensation, subjects might not be able to perform the task with the same facility. An additional research question is how subjects perform in this task when they use two hands. Van der Horst and Kappers (2007) studied the discrimination performance of convex shapes in a unimanual and in a bimanual mode, and found a higher threshold in the latter case. As can be seen in figure 2, in unimanual modes, congruent shapes are oriented in the same direction with respect to the external space, whereas opponent shapes are oriented oppositely. In contrast, in bimanual modes, congruent shapes can be oriented oppositely, whereas opponent shapes have the same direction with respect to the external space.

The aim of this paper is to explore how human observers haptically discriminate object surfaces that are described by the same physical curvature but vary in the orientation with respect to the fingers and with respect to external space. We investigated this in a series of experiments. We were interested in what respect the perception 


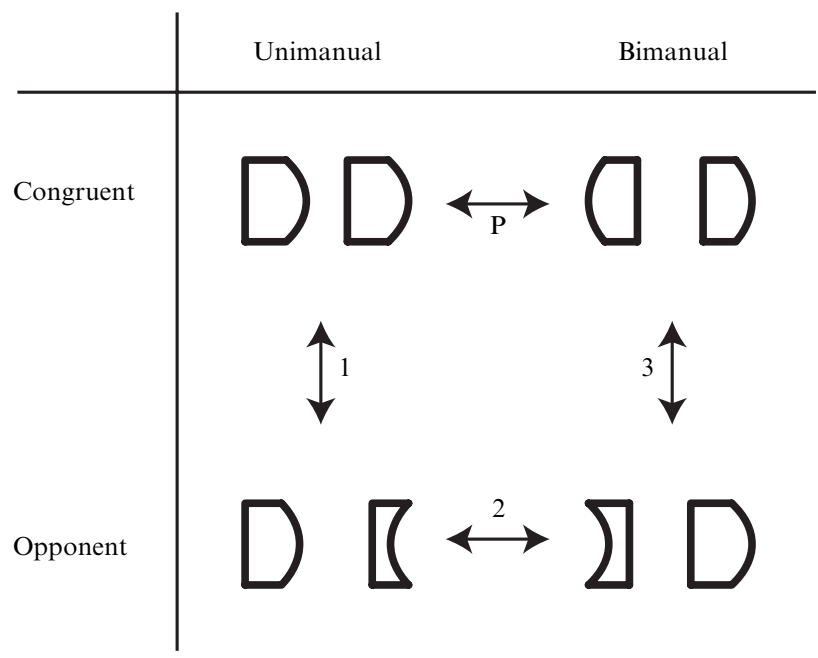

Figure 2. Scheme of the experiments conducted in this study. The numbers refer to the experiments. 1. Curvature-discrimination performance was studied for congruent modes and opponent modes in a unimanual condition. The experiment was conducted for strongly curved stimuli and for weakly curved stimuli. 2. Curvature-discrimination performance was compared for a unimanual mode and bimanual modes. Opponently curved shapes were used. A similar experiment has already been conducted by van der Horst and Kappers (2007) for congruent shapes (P). 3. Comparison of curvature-discrimination performance for congruent shapes and opponent shapes in a bimanual condition.

of opponent shapes differs from the perception of congruent shapes and why this difference occurs. We studied the influence of factors such as the degree of curvature of the surfaces, the orientation of the stimuli with respect to the finger and to the external space, and the use of one hand or two hands. An overview of the experiments is given in figure 2 .

\section{Experiment 1}

In experiment 1, we studied whether subjects can discriminate the curvature of opponent shapes with the same precision as they can discriminate the curvature of congruent shapes. Two parameters can be distinguished: the sensitivity of a subject (discrimination thresholds) and the point of subjective equality (bias). One would expect similar thresholds for the opponent mode and the congruent modes, since the slope difference and the curvature are the important cues in the curvature discrimination of congruent shapes. However, were a higher threshold to be found in the opponent mode, this would indicate that not only the slope difference and curvature, but also the direction of the curved surface, are important in shape perception. The second parameter, the bias, represents the curvature difference between two stimuli which were on average judged to be equal.

The experiments in this study were primarily performed with strongly curved surfaces (the curvatures used are similar to the curvature of a coffee cup). However, we decided to measure in addition the performance for weakly curved stimuli (the curvatures of weakly curved stimuli are similar to the curvature of a water butt). For weakly curved stimuli, we know already that the discrimination performance is similar for convex and concave stimuli (Goodwin et al 1991; Kappers and Koenderink 1996; Pont et al 1997; Louw et al 2000). When the slope difference and curvature are the only cues that subjects use, no differences between the congruent modes of the strongly curved stimuli are to be expected. However, since the index finger itself has a convex shape, the contact area of the finger with a concave surface is larger than the contact area of the finger with a convex surface, and, what is more, a shorter movement 
suffices to explore the whole stimulus surface. These differences in contact area and scanning length are probably negligible for long, weakly curved stimuli, but not for short, strongly curved stimuli. We hypothesise that, when strongly curved stimuli are used, a concave stimulus induces a stronger sense of curvature than a convex stimulus, although their physical curvature is identical. As a first consequence, lower thresholds might be obtained in the concave-concave mode than in the convex-convex mode, whereas similar thresholds are obtained for the weakly curved stimuli. Second, a systematic bias should occur when subjects have to compare the curvature of two strongly curved opponent shapes, but no systematic bias should occur when the weakly curved stimuli are used.

\subsection{Method}

2.1.1 Subjects. Six subjects participated (three female and three male; mean age 25 years). They were paid for their efforts and they were naive with respect to the design of the experiment. A standard questionnaire established that all subjects were strongly righthanded (Coren 1993).

2.1.2 Stimuli. Convex and concave stimuli with a constant curvature were used. A distinction was made between strongly curved stimuli and weakly curved stimuli. The strongly curved stimuli were made of a compound of polyurethane foam and artificial resin (Cibatool BM 5460). The curvature of the test stimuli varied from $\pm 20 \mathrm{~m}^{-1}$ to $\pm 46 \mathrm{~m}^{-1}$, in steps of $1 \mathrm{~m}^{-1}$. The curvature values of the reference stimuli were $\pm 33 \mathrm{~m}^{-1}$. The convex stimuli had already been used by van der Horst and Kappers (2007). The weakly curved stimuli were made of PVC. The curvature of these test stimuli varied from $\pm 0.2 \mathrm{~m}^{-1}$ to $\pm 3.8 \mathrm{~m}^{-1}$, in steps of $0.2 \mathrm{~m}^{-1}$. The curvatures of the reference stimuli were $\pm 2.0 \mathrm{~m}^{-1}$. This stimulus set had already been used by Pont et al $(1998,1999)$. Schematic illustrations of the stimuli are given in figures 3a-3d. Although the materials of the weakly and strongly curved stimuli were different, their surfaces were both very smooth. We presented the stimuli in the same orientation as in the previous studies.

2.1.3 Design and procedure. 6 conditions were studied in a 3 (mode) $\times 2$ (curvature) repeated-measures design. In the opponent mode, convex shapes were compared to concave shapes; in the congruent modes, either convex or concave stimuli were used. All three modes were tested for both strongly curved stimuli and for weakly curved stimuli.

Subjects were seated behind a curtain. Their forearm rested on a table. They put their right hand under the curtain to touch the stimuli without seeing them. The stimuli were presented in front of the subjects, $40 \mathrm{~cm}$ from the edge of the table. The strongly curved stimuli were placed on a stand; the weakly curved stimuli were placed in a holder on the table. Subjects explored the stimuli by passing the distal phalanx of their index finger to and fro over the stimulus surface. The axis of the finger was oriented so that it was perpendicular to the curvature.

Discrimination experiments were conducted by means of a two-alternative forcedchoice (2AFC) procedure. In each trial, subjects had to indicate which of the two sequentially presented stimuli felt the more curved. No restrictions were imposed on the number of movements over the stimulus or on the number of alternations between the stimuli. ${ }^{(1)}$ No feedback was provided to subjects regarding their performance.

In each condition, stimulus combinations of ten values of curvature difference were chosen, as specified below. Curvature difference is defined as the difference in the absolute curvature between either the convex and the concave stimulus (opponent mode)

(1) Extensive pilot experiments showed that the enforcement of restrictions resulted in a wider variance between subjects. 
Experiment 1

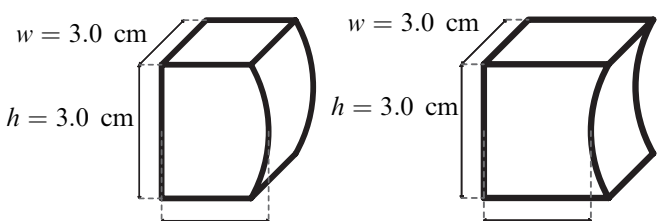

(a) $l=2.4 \mathrm{~cm}$

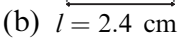

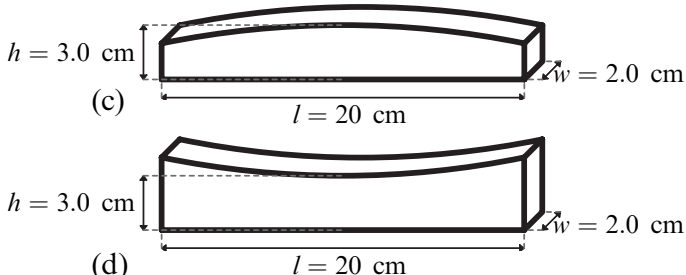

Experiment 2

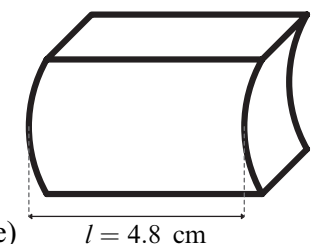

Experiment 3

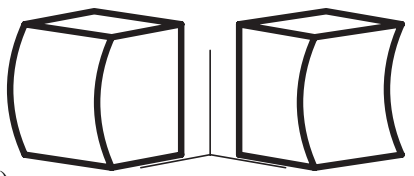

(f)

Figure 3. Schematic drawings of the stimuli used in this study. Experiment 1: (a) strongly curved convex stimulus, (b) strongly curved concave stimulus, (c) weakly curved convex stimulus, (d) weakly curved concave stimulus. Notice the difference in scale between the strongly curved stimuli and the weakly curved stimuli. The curvature of the strongly curved stimuli was $33 \mathrm{~m}^{-1}$. The curvature of the weakly curved stimuli was $2.0 \mathrm{~m}^{-1}$. The strongly curved stimuli were placed on a stand, $5 \mathrm{~cm}$ above the table top. The weakly curved stimuli were placed in a holder, directly on the table top. Subjects moved the tip of the index finger either upwards and downwards (strongly curved stimuli) or leftwards and rightwards (weakly curved stimuli) over the stimulus surface. Experiment 2: (e) example of a stimulus combination used for a bimanual condition. The surfaces were similar to the strongly curved surfaces of experiment 1 . One index finger explored the convex surface, the other index finger explored the concave surface. Experiment 3: (f) example of a stimulus combination of two opponent shapes. The surfaces were similar to the strongly curved surfaces of the preceding experiments. The angle between the orientations of the stimuli is $90^{\circ}$. The horizontal distance between the middle parts of the two surfaces is $10 \mathrm{~cm}$. Notice that the drawing depicts the backs of the stimuli. In this example, the convex surface was explored by the right index finger and the concave surface was explored by the left index finger.

or the first and the second stimulus presented (congruent modes). In the opponent mode, we did not know in advance whether biases would occur, and, if they occurred, we did not know whether they would differ between subjects. Hence, before beginning the actual experiment, we obtained an estimate of the bias level. We did this by performing a staircase experiment, with a simple up-down method (Treutwein 1995). Each trial in a sequence was determined by the outcome of the preceding trial in that sequence. Starting at two different curvature-difference values, two sequences, each consisting of 15 trials, were randomly interleaved. The mean curvature-difference value in the outcome of the last 8 trials of both sequences was taken as the estimated bias level. The stimuli were chosen around this estimated bias level. This level was restricted to $\pm 4 \mathrm{~m}^{-1}$ for the strongly curved stimuli and to $\pm 0.4 \mathrm{~m}^{-1}$ for the weakly curved stimuli. In contrast to our expectations for the opponent mode, we did not expect biases to occur in the congruent modes. Therefore, the stimuli were chosen symmetrically around a zero level of curvature difference. The curvature differences of the stimuli with respect to the estimated bias level were $\pm 1,3,5,7,9 \mathrm{~m}^{-1}$ for the strongly curved stimuli, and $\pm 0.2,0.4,0.6,1.0,1.4 \mathrm{~m}^{-1}$ for the weakly curved stimuli, respectively.

Each condition consisted of 120 trials (10 curvature-difference values $\times 12$ repetitions), which were presented in a pseudo-random order: groups of all possible stimulus combinations were each randomised and presented successively. In one session, only a single condition was measured. Half of the subjects first performed the conditions with the strongly curved stimuli, whereas the remaining subjects started with the weakly curved stimuli. The order in which the modes were executed was counterbalanced among subjects. It took about $6 \mathrm{~h}$ per subject to complete the whole experiment. 
2.1.4 Analysis. For each subject-condition combination, the fraction of responses was expressed as a function of curvature difference. Psychometric functions (cumulative Gaussians) were fitted to these data by means of maximum-likelihood procedure. Examples of data sets and the accompanying psychometric functions are given in figure 4. The psychometric function is characterised by the threshold $\sigma$ and the bias $\mu$. The bias is the point of subjective equality. A positive bias value in the opponent mode means that the curvature of a more convex surface is on average judged to be equal to the curvature of a less concave surface. The threshold is defined as the curvature difference between the 84\%-point and the bias level (50\%-point). In order to establish the goodness of fit for each individual fitted curve, we used the method described by Wichmann and Hill (2001a). The deviance of the measured data set from the fitted curve is determined and compared to a distribution of deviances of 10000 simulated data sets, obtained by a parametric bootstrap technique. The goodness of fit is poor when the deviance of the measured data set is higher than the 0.975 point of the percentile confidence interval of the distribution of deviances. The parametric bootstrap method was also used to obtain an estimate of the confidence level of each individual threshold and bias level (see Wichmann and Hill 2001b).
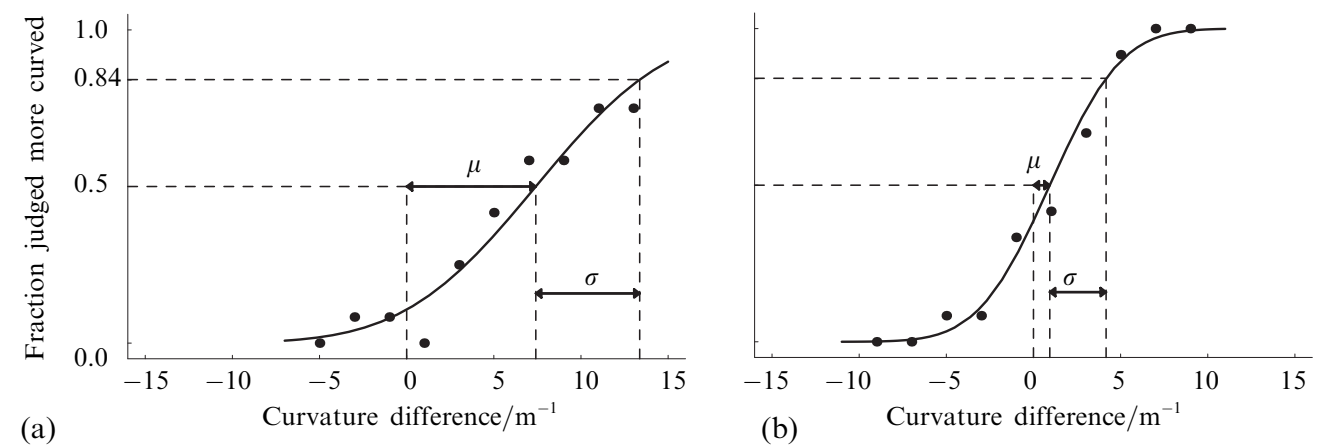

Figure 4. Examples of psychometric curves for strongly curved stimuli in (a) the opponent mode (convex - concave conditions) and (b) the congruent mode (concave-concave conditions). Curvature difference is defined as the difference in the absolute curvature of either the convex and the concave stimulus (opponent mode) or the first and the second stimulus presented (congruent mode). The discrimination threshold is represented by $\sigma$; it is a measure of the sensitivity of subjects. The bias is represented by $\mu$; it is the difference in the curvature of two stimuli that were judged to be equally curved. Biases occurred in the opponent modes, but were near zero in the congruent modes.

\subsection{Results}

Figures $5 \mathrm{a}$ and $5 \mathrm{~b}$ show the mean threshold values for all conditions. The error bars indicate the standard error for each condition without any correction for the variability in the mean thresholds for subjects. The thresholds were higher for the opponent modes than for the corresponding congruent modes; the thresholds for the congruent modes were comparable. The significance of the results was tested by a 3 (mode) $\times$ 2 (curvature) ANOVA with a repeated-measures design. First, a significant main effect was found for mode $\left(F_{2,10}=20.3, p<0.001\right)$. As a further analysis we performed a planned comparison between, on the one hand, the opponent mode, and, on the other hand, the congruent modes. The contrast of the opponent mode versus the congruent modes was significant $\left(F_{1,5}=27.6, p=0.003\right)$, but the contrast between the congruent modes (convex-convex versus concave-concave) was not significant $\left(F_{1,5}=3.2\right.$, $p=0.1)$. When repeated-measures ANOVAs were performed for the two curvature regimes separately, the results were in accordance with the former analysis: the main effect was significant for the strongly curved stimuli $\left(F_{2,10}=21.5, p<0.001\right)$ and for the weakly curved stimuli $\left(F_{2,10}=5.2, p=0.028\right)$; the contrasts between the opponent 
(a)
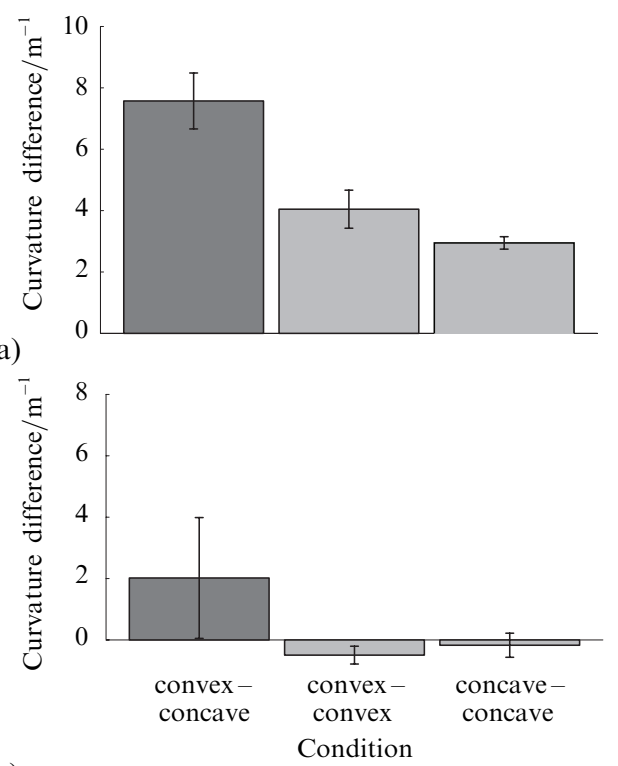

(c) (b)
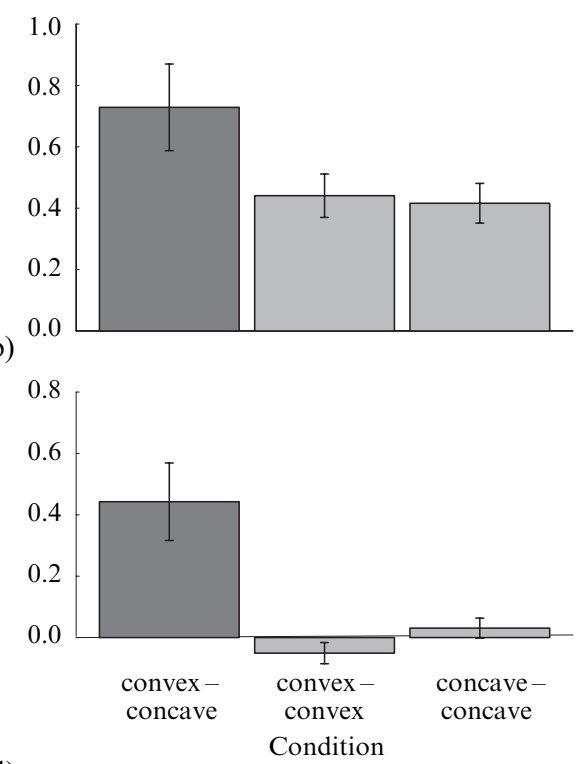

(d)

Figure 5. Results of experiment 1 for (a) and (b) threshold and (c) and (d) bias. Each bar represents the mean value for six subjects in the specific condition. Note the difference in scale between the strongly curved stimuli in (a) and (c) and the weakly curved stimuli in (b) and (d). The error bars indicated are the standard error of the mean for each condition.

mode and the congruent modes were also significant $\left(F_{1,5}=30.0, p=0.003\right.$ and $F_{1,5}=7.2, p=0.043$, respectively), but no significant difference was found between the congruent modes $\left(F_{1,5}=3.5, p=0.1\right.$ and $F_{1,5}=0.09, p=0.8$, respectively). Second, a main effect of curvature was found $\left(F_{1,5}=99.8, p<0.001\right)$. This reflects the fact that the thresholds were higher for the strongly curved stimuli than for the weakly curved stimuli. Third, the interaction of mode and curvature appeared to be significant $\left(F_{2,10}=22.4, p<0.001\right)$. This might, however, be ascribed to the effect of curvature, since no interaction effect was found when the ANOVA was performed on normalised data $\left(F_{2,10}=2.9, p=0.1\right)$.

The results for the biases are shown in figures $5 \mathrm{c}$ and $5 \mathrm{~d}$. The biases were on average positive for the opponent modes and near zero for the congruent modes. In the opponent modes, strong differences were found between subjects, as the error bars manifest. A $t$-test performed on each separate condition showed only a significant difference from zero for the convex-concave mode of the weakly curved stimuli $\left(t_{5}=3.5, p=0.017\right)$. We also looked at the significance of individual biases for all subjects in each condition by comparing a bias value to an estimate of the confidence level. In the opponent mode, the bias was significant for four out of six subjects when the strongly curved stimuli were used and for five subjects when the weakly curved stimuli were used. For the congruent stimuli, only one significant bias was found in each condition.

The goodness of fit was tested for all 36 curves. With only one exception, all fitted curves were judged to be good. So we can be confident that our data are well described by the fits of the psychometric curves.

\subsection{Discussion}

This experiment revealed that discriminating the curvature of a convex shape from a concave shape is more difficult than discriminating the curvature of either a convex shape from a convex shape or a concave shape from a concave shape. No differences were found between the convex-convex mode and the concave-concave mode. 
The threshold results for the convex-convex conditions can quantitatively be compared with the results of previous studies. First, the results for the weakly curved stimuli reproduced those of Pont et al (1999), who found threshold values of $0.4 \mathrm{~m}^{-1}$. Second, the results for the strongly curved stimuli were comparable to those of van der Horst and Kappers (2007), who obtained $2.3 \pm 0.4 \mathrm{~m}^{-1}$ and $3.3 \pm 0.8 \mathrm{~m}^{-1}$ in different experiments. Finally, when the stimuli and thresholds are recalculated in stimulus widths and height differences, they were consistent with Louw et al (2002b).

The similarity in the thresholds for the congruent modes was found not only for the weakly curved stimuli, but also for the strongly curved stimuli. The former is in agreement with previous research (Goodwin et al 1991; Kappers and Koenderink 1996; Pont et al 1997; Louw et al 2000). For the latter, we had suggested that subjects would obtain a stronger sense of curvature from a concave surface than from a convex surface, but we did not find this effect in our data. The results for the congruent conditions confirm that the curvature discrimination of convex from convex and concave from concave shapes is based on the same cues, which we suppose to be mainly the slope difference between the stimuli, and, to a lesser extent, the curvature (Pont et al 1997, 1999; Louw et al 2002a, 2002b).

The higher thresholds obtained in the opposite mode than in the congruent modes rule out the possibility that the discrimination of two opponent surfaces can simply be reduced to the cues important for the congruent modes, namely the slope difference and the curvature. Moreover, wrong biases were found, which were on average positive. A positive bias means that subjects systematically underestimated the curvature of a convex stimulus compared to the curvature of a concave stimulus. This is consistent with the hypothesis that subjects might have obtained a stronger sensation from a concave stimulus than from a convex stimulus, owing to a difference in contact area of the finger with the stimuli and a difference in scanning length of the finger over the stimulus surface. Although this hypothesis was originally formulated for the strongly curved stimuli and not for the weakly curved stimuli, we found only a significant effect for the weakly curved stimuli. However, the size of the biases we found differed to a great extent between subjects. Still, differences in contact area and scanning length might have played a role, but are certainly not the only factors that contributed to the biases we found.

One might wonder whether the difference in orientation between the strongly curved stimuli and the weakly curved stimuli might have influenced our results. However, the striking similarity in the results of the strongly curved stimuli and the weakly curved stimuli is a strong indication that this is not the case. Moreover, Pont et al (1998) already showed that the size of the threshold was not affected by the orientation of the stimuli with respect to the external space. Therefore, we have no reason to expect that the results depend on the horizontal or vertical orientation of the stimuli.

The higher thresholds obtained in the opponent mode compared to the congruent mode and the occurrence of biases in the opponent mode might be related to the fact that, in the opponent mode, subjects had to compare the curvature of surfaces with an opposite direction with respect to the finger and with respect to the external space. Oldfield and Phillips (1983) observed that the perceived orientation of a raised letter that indented the skin of the finger depended on the orientation of the finger and the body with respect to the external space. They suggested that subjects do not process skin indentation directly, but map tactile impressions onto a system of external references. When subjects had to discriminate same-shaped and mirror-shaped stimuli, they achieved faster reaction times and higher accuracy with the former, irrespective of whether mental rotation was required (Dellantonio and Spagnolo 1990; Prather and Sathian 2002). We can imagine that the mental process of curvature comparison is more complex for opponent shapes than for congruent shapes. In a congruent condition, 
subjects might be able to reduce their sensation directly to a slope difference and a curvature difference, whereas, in an opponent condition, they have to perform mental alignment operations, such as mental rotation or mirroring, before they are able to compare the slope difference and the curvature difference. However, another possibility is that subjects have difficulty in distinguishing signals from opposite shapes, obtained with one and the same finger. We developed experiments 2 and 3 to address these issues.

\section{Experiment 2}

In all conditions of experiment 1 , subjects used a single finger to explore each stimulus pair. In experiment 2, we compared the mode in which subjects used a single finger (unimanual mode) to the mode in which subjects used two index fingers, one finger per stimulus (bimanual mode). Previously, van der Horst and Kappers (2007) conducted a similar experiment involving congruent (convex) surfaces. They found higher thresholds in the bimanual condition than in the unimanual condition - the difference being attributed to the fact that in a bimanual condition, signals have to be assembled from different fingers.

In the current experiment, only opponent stimulus combinations were used. The results of van der Horst and Kappers (2007) supported the expectation that higher thresholds might be found in the bimanual mode. However, an advantage in this mode might be that the opponent surfaces are not sensed by the same finger. Subjects might also benefit from the fact that, in the bimanual mode, the surfaces are parallel in the external space, as can be seen in figure $3 \mathrm{e}$.

\subsection{Method}

Twelve right-handed subjects (five female and seven male, mean age 23 years), who were not involved in experiment 1, participated. They were paid for their efforts and they were naive with respect to the design of the study. The strongly curved stimuli of experiment 1 were used. Three conditions were studied: one unimanual condition and two bimanual conditions. The unimanual condition was identical to the opponent condition of experiment 1: a convex and a concave stimulus were presented successively to the right index finger. In the bimanual conditions, the convex surface was presented to the index finger of the right hand and the concave surface was presented to the index finger of the left hand, or vice versa. Figure $3 \mathrm{e}$ shows a stimulus in a bimanual condition. The distance between the middle points of the left and right surfaces was $4.8 \mathrm{~cm}$. The procedure was similar to that of experiment 1 . No restrictions were imposed on the exploration time or on the number of explorations of each stimulus, but subjects were not allowed to explore two stimuli simultaneously. The number of trials and the stimulus choice procedure were identical to the opponent condition of the strongly curved stimuli in experiment 1 . For each subject, the first session was devoted to obtaining an estimate of the bias level in each condition. In the subsequent three sessions, the actual experiment was performed in which the data for the psychometric curves were obtained. The order in which subjects completed the experiment was counterbalanced. Each subject took 3-4 h to complete the whole experiment.

\subsection{Results}

The results are shown in figure 6. On average, a slightly higher threshold value was found in the unimanual condition than in the bimanual conditions. However, the ANOVA with a repeated-measures design showed no significant main effect $\left(F_{1.3,13.7}=3.4, p=0.08\right.$, $\varepsilon=0.625)$. The degrees of freedom were corrected by a Greenhouse-Geiser $\varepsilon$ correction.

The biases were on average positive. In each condition, a significant deviation from zero was found, as $t$-tests confirmed $\left(t_{11}=2.7, p=0.019\right.$ for the unimanual condition, and $t_{11}=4.5, p=0.001$ and $t_{11}=3.6, p=0.004$ for the two bimanual conditions, respectively).

Each individual psychometric curve was judged to be good, according to the goodnessof-fit test. 

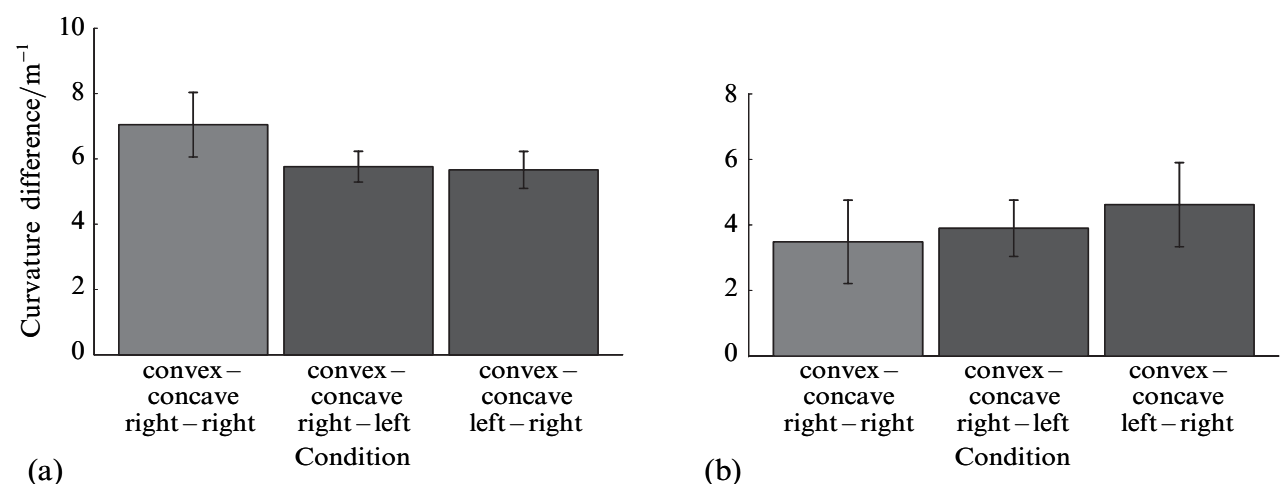

Figure 6. Results of experiment 2 for (a) threshold and (b) bias. Each bar represents the mean value for twelve subjects in the specific condition.

\subsection{Discussion}

Unlike the results of van der Horst and Kappers (2007), who found an increase in threshold from the unimanual condition to the bimanual condition, the current experiment showed no increase in threshold from the unimanual mode to the bimanual mode. Nevertheless, in the previous study congruent stimuli were used, whereas in this experiment we used opponent stimuli. Apparently, the disadvantage of a bimanual comparison (assemblage of signals from different fingers) compared to a unimanual comparison was countered by some advantageous factors, such as the parallelity of the surfaces in the external space, or the fact that the fingers did not have to alternate between a convex surface and a concave surface. To study this more thoroughly, we performed experiment 3 .

No differences in threshold were found between the two bimanual conditions. This is in agreement with previous results, ie no differences were found in the ability to discriminate the curvature of only convex surfaces or concave surfaces (first experiment) or the ability to discriminate curvature with only the right index finger or the left index finger (van der Horst and Kappers 2007).

Significant, positive biases were found in each condition. Although the size of the bias varied again from subject to subject, the mean bias was clearly positive.

\section{Experiment 3}

In experiment 3 , subjects bimanually discriminated the curvature in two modes-one with opponent shapes, the other with congruent shapes. To rule out any advantage of the orientation of the stimuli in external space, we placed the stimuli at perpendicular orientations (see figure 3f). If the orientation of the surfaces is important and subjects have more difficulty in comparing the curvature of opponent shapes than that of congruent shapes, a higher threshold might be expected for the former comparison. Otherwise, results might be similar.

\subsection{Method}

Six paid, right-handed subjects (five female and one male, mean age 23 years) participated. ${ }^{(2)}$ They were naive with respect to the design of the experiment and were not involved in the previous experiments. The strongly curved stimuli were used. Two bimanual conditions were studied. In one condition, a convex surface was explored by the right index finger and a concave surface was explored by the left index finger.

(2) We had to exclude another subject (male, 59 years old) from the experiment. For this subject, the preliminary experiment conducted to obtain an estimate of the bias revealed a bias that was far outside our measurement range. 
In the other condition, only convex surfaces were used for both index fingers. The stimuli were placed $40 \mathrm{~cm}$ from the edge of the table. The distance between the middle points of the left and right surfaces was $10 \mathrm{~cm}$. The angle between the imaginary normals at the middle points was $90^{\circ}$. Figure $3 \mathrm{f}$ shows an example of the stimuli for the opponent mode. The procedure, the number of trials, and the stimulus choice were similar to the procedure followed in previous experiments. The measurement of a single condition (estimate of the bias and the actual experiment) was performed in a single session. The order in which subjects completed the experiment was counterbalanced. Each subject took about $2 \mathrm{~h}$ to complete the whole experiment.

\subsection{Results}

Figure 7 shows the results. The results for the thresholds were similar. A two-tailed $t$-test confirmed that there was no significant effect $\left(t_{5}=0.28, p=0.8\right)$. The biases were on average positive, but, for each condition, did not differ significantly from zero $\left(t_{5}=2.2, p=0.084\right.$ for the opponent condition and $t_{5}=2.3, p=0.068$ for the congruent condition). However, significant biases were found for individual subject-condition combinations (five for the opponent condition and three for the congruent condition). The assessment of the goodness-of-fit test showed that each individual psychometric curve was judged to be good.

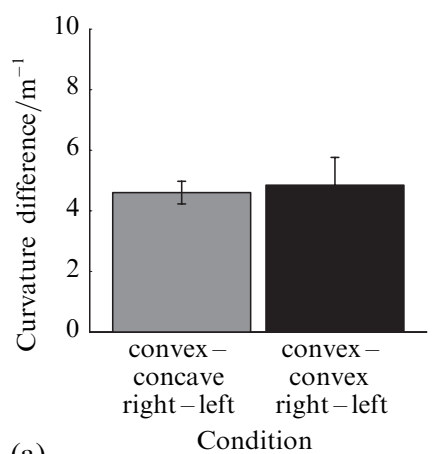

(a)
Condition

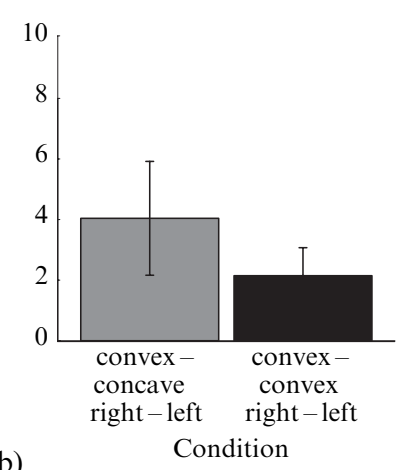

(b)

Figure 7. Results of experiment 3 for (a) threshold, (b) bias. Each bar represents the mean value for six subjects in the specific condition.

As an additional analysis we performed a comparison between, on the one hand, the threshold results of this experiment and, on the other hand, the results of two conditions of experiment 1: the opponent condition and the convex-convex condition of the strongly curved stimuli. A $2 \times 2$ ANOVA was conducted with shape (opponent versus congruent) as a within-subjects factor and hands (unimanual versus bimanual) as a between-subjects factor. A significant main effect was found for shape $\left(F_{1,10}=8.7\right.$, $p=0.015)$ and for the interaction between shape and hands $\left(F_{1,10}=21.3, p=0.007\right)$, but not for hands $\left(F_{1,10}=1.5, p=0.3\right)$.

\subsection{Discussion}

This experiment showed clearly that the curvature-discrimination performance in a bimanual situation did not depend on whether congruent shapes or opponent shapes were used. The significant interaction between shape and hands confirmed that this result is in contrast to the unimanual results of the first experiment, where we found higher thresholds for the opponent shapes than for the congruent shapes.

The results for the biases in the opponent condition are in accordance with the previous experiments. Subject-dependent biases were obtained in the congruent condition, as van der Horst and Kappers (2007) already showed, when they measured this condition. 


\section{General discussion}

\subsection{Threshold}

We studied human performance of haptic curvature discrimination using opponent shapes and congruent shapes. When subjects used only a single index finger, a clearly higher threshold was found for the opponent mode than for the congruent mode (experiment 1). However, similar thresholds for the opponent mode and the congruent mode were obtained when subjects used two index fingers, one finger per stimulus surface (experiment 3). When the unimanual mode and the bimanual mode for the opponent shapes were directly compared, a slightly higher threshold was found in the unimanual mode, although this was not significant. However, significant or not, the important message is that this finding is different from previous research on the curvature discrimination of congruent shapes, where higher thresholds were found in the bimanual mode (van der Horst and Kappers 2007).

Previous research has demonstrated that the slope difference and the curvature are the important cues in curvature discrimination in a congruent, unimanual mode (Pont et al 1997, 1999; Louw et al 2002a, 2002b). The results of the congruent conditions we measured in experiment 1 were in agreement with this concept, since we observed similar thresholds for the convex-convex mode and for the concave-concave mode. The fact that we found significantly higher thresholds in the opponent mode of experiment 1 does not necessarily mean that the slope difference and the curvature are not important cues in this mode, but probably indicates that subjects were not able to use these cues directly. In section 2.3, we hypothesised that mental alignment processes such as rotation or mirroring, or the orientation of the stimuli with respect to the fingers and the external space, made the curvature-discrimination task more difficult with opponent shapes than with congruent shapes. However, experiment 3 showed that the curvature-discrimination performance did not depend on whether opponent or congruent shapes were used when these surfaces were presented bimanually. This excluded a contributing factor of the orientation of the stimuli in the external space and made the assumption that mental alignment processes were involved in the comparison of opponent stimuli superfluous for the bimanual case. This leaves the question whether the unimanual curvature discrimination of opponent stimuli was performed by these processes. A clear difference between the unimanual opponent mode and the other modes we have studied is that in the unimanual opponent mode opponent surfaces were sensed by the same finger, whereas in the unimanual congruent conditions and the bimanual opponent conditions this was not the case. We can imagine that the mental processing of opposite signals from a single finger is more complex to perform than the processing of congruent signals from a single finger. Probably, this processing of opposite signals was performed bilaterally, just like the bimanually obtained signals. Recently performed brain-imaging studies have shown that, dependent on the task, unimanual tasks are processed unilaterally (Roland et al 1998), bilaterally (Zhang et al 2005), or dominantly in one hemisphere (Van Boven et al 2005). A disadvantage of bilateral processing is that the information is degraded by interhemispheric transmission, but an advantage is that the task load is shared between both hemispheres (Bradshaw et al 1998). Of course, we cannot prove that the unimanual opponent signals have been processed bilaterally, but it is a possible explanation for the obtained results and makes the mental alignment hypothesis redundant.

\subsection{Bias}

Biases were found in all modes in which either opponent shapes or two hands were involved. Concerning the opponent shapes, significant, positive biases were found for the weakly curved condition of experiment 1 and for all three conditions of experiment 2. A nearly significant, positive bias was obtained in experiment 3 . 
(Remember, a positive bias means that the curvature of a convex shape is systematically underestimated compared to the curvature of a concave shape.) A further observation with respect to the biases is that the size of the bias differed strongly among subjects and conditions, which is in accordance with previous bimanual curvaturediscrimination studies (Kappers and Koenderink 1996; Sanders and Kappers 2006; van der Horst and Kappers 2007). We suggest that the cause of the bias is twofold. The idiosyncratic part of the bias might result from the rather complex mental process of comparing bimanual or opponent signals. We suppose that the positive part of the bias originates from the manner in which the surfaces of the stimuli are explored. As the finger itself has a convex shape, a larger part of the finger is at each time in contact with a concave stimulus than with a convex stimulus of the same curvature, on the assumption that the stimulus is touched with an equal amount of force. However, in the experiments we performed, the finger is not in static contact with the stimulus surface, but moves over the stimulus surface. Because of the shape of the finger itself, a shorter exploration distance and time suffices to explore the whole stimulus surface of a concave stimulus than that of a convex stimulus. Finally, the finger can be rotated when it is moved over the stimulus surface. The question that arises is how the way of exploration might have caused the biases we found.

Recently, Hayward (2008) described how the contact point between the finger and the stimulus surface shifts when the finger makes a rightward movement over the stimulus surface, by either rolling or sliding. For a convex surface, rolling shifts the contact point rightwards and sliding shifts the contact point leftwards; for a concave surface, both rolling and sliding shift the contact point rightwards (figures 8a, 8b, 8d, 8e). Now the question is: did the subjects in our experiments perform rolling or sliding movements, or was the movement pattern different? Informal observations of the exploration strategy of our subjects showed that subjects tended to keep the finger in such a way that the fingernail was parallel to the tangent plane of the contact surface. On convex surfaces, this was achieved by a combination of rolling and sliding (figure 8c). However, on a concave surface, this could not be realised by a combination of rolling and sliding, but was achieved by a combination of sliding and rotation, but, importantly, a rotation in the opposite direction to rolling (figure 8f). This type of movement differs from that described by Hayward for concave surfaces.

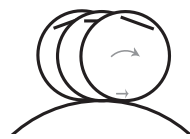

(a)

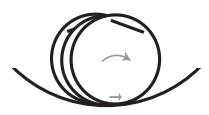

(d)

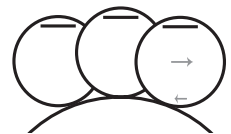

(b)

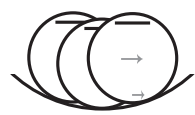

(e)

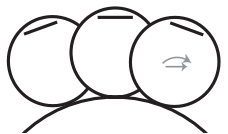

(c)

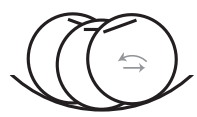

(f)

Figure 8. Movement profiles of different finger explorations of a rightward movement on a convex [(a), (b), and (c)], and a concave [(d), (e), and (f)] surface. Inspiration for (a), (b), (d), (e) has been derived from figure 3 in Hayward (2008). When the finger rolls over the surface [(a) and (d)], the contact point of the finger with the surface shifts rightwards. When the finger slides over the surface, the contact point shifts either leftwards on a convex surface (b) or rightwards on a concave surface (d). As subjects tended to keep their finger in such a way that the contact point did not shift, the movement of the finger on a convex surface resulted from a combination of rolling and sliding (c). However, on a concave surface, the movement resulted from a combination of rotation and sliding, but the rotation was in the opposite direction to rolling (f). 
What might this difference in exploration mean for the ways in which the curvatures of the convex surface and concave surface are perceived? From mechanics, it is known that the friction force that a surface exerts on a circular object like a wheel is lower when this object rolls than when it slides over the surface. Applied to our setup, the friction force that a convex surface exerts on the finger consists of a roll friction component and a slide friction component. This total friction force is lower than when the finger would only slide over the surface. The force that a concave surface exerts on the finger consists of a slide friction component of the translation with, in addition, a slide friction component of the rotation. For this case, the total friction force is higher than when the finger would slide over the surface, without rotation. This means that the friction exerted by a concave surface is higher than the friction exerted by a convex surface. This might explain why the curvature of the convex surface is underestimated compared to the curvature of a concave surface, as it has been shown that the sensation of a larger force can be interpreted as a higher perceived curvature (Robles-de-la-Torre and Hayward 2001; Drewing and Ernst 2006). Future studies might test the validity of this explanation by further disentangling how exploration differences in scanning length and time, in rolling and rotational movement, and in exerted force and pressure, contribute to this interesting finding.

\section{Conclusion}

When the ability to discriminate the curvature of opponent shapes was compared to the ability to discriminate the curvature of congruent shapes, a significant decrease in performance was found when a single finger was used to explore both surfaces, but similar results were obtained when different fingers touched each surface. The result for the bimanual mode shows that a congruent or incongruent orientation of the surfaces with respect to the fingers or with respect to the external space does not necessarily influence the sensitivity to perform the task. The decrease in performance in the unimanual mode might be due to the difficulty of processing opposite signals from a single finger.

Humans are systematically biased when they have to compare the curvature of opponent shapes, either unimanually or bimanually. This means that the curvature of convex shapes is generally underestimated compared to the curvature of concave shapes. We suggest that these biases result from the manner in which convex and concave shapes are explored.

Acknowledgment. This research was supported by a grant from the Netherlands Organisation for Scientific Research (NWO).

\section{References}

Bradshaw J L, Nicholls M E R, Rogers M A, 1998 “An intermanual advantage for tactual matching” Cortex $34763-770$

Coren S, 1993 The Left-handed Syndrome (New York: Vintage Books)

Dellantonio A, Spagnolo F, 1990 "Mental rotation of tactual stimuli" Acta Psychologica 73 $245-257$

Drewing K, Ernst M O, 2006 "Integration of force and position cues for shape perception through active touch" Brain Research $107892-100$

Goodwin A W, John K T, Marceglia A H, 1991 "Tactile discrimination of curvature by humans using only cutaneous information from the fingerpads" Experimental Brain Research 86 $663-672$

Gordon I E, Morison V, 1982 "The haptic perception of curvature" Perception \& Psychophysics $31446-450$

Hayward V, 2008 "Haptic shape cues, invariants, priors, and interface designs", in Human Haptic Perception: Basics and Applications Ed. M Grunwald (Basel: Birkhäuser Verlag)

Horst B J van der, Kappers A M L, 2007 "Curvature discrimination in various finger conditions" Experimental Brain Research $177304-311$ 
Kappers A M L, Koenderink J J, 1996 "Haptic unilateral and bilateral discrimination of curved surfaces" Perception $25739-749$

LaMotte R H, Srinivasan M A, 1996 "Neural coding of shape: responses of cutaneous mechanoreceptors to wavy surfaces stroked across the monkey fingerpad" Journal of Neurophysiology $763787-3797$

Louw S, Kappers A M L, Koenderink J J, 2000 "Haptic detection thresholds of Gaussian profiles over the whole range of scales" Experimental Brain Research 132369 - 374

Louw S, Kappers A M L, Koenderink J J, 2002a "Active haptic detection and discrimination of shape" Perception \& Psychophysics $641108-1119$

Louw S, Kappers A M L, Koenderink J J, 2002b "Haptic discrimination of stimuli varying in amplitude and width" Experimental Brain Research 14632 - 37

Oldfield S R, Phillips J R, 1983 "The spatial characteristics of tactile form perception” Perception $12615-626$

Pont S C, Kappers A M L, Koenderink J J, 1997 "Haptic curvature discrimination at several regions of the hand" Perception \& Psychophysics 59 1225-1240

Pont S C, Kappers A M L, Koenderink J J, 1998 "The influence of stimulus tilt on haptic curvature matching and discrimination by dynamic touch" Perception $27869-880$

Pont S C, Kappers A M L, Koenderink J J, 1999 "Similar mechanisms underlie curvature comparison by static and dynamic touch" Perception \& Psychophysics 61 874-894

Prather S C, Sathian K, 2002 "Mental rotation of tactile stimuli" Cognitive Brain Research 14 91 - 98

Robles-de-la-Torre G, Hayward V, 2001 "Force can overcome object geometry in the perception of shape through active touch" Nature $412445-448$

Roland P E, O'Sullivan B, Kawashima R, 1998 "Shape and roughness activate different somatosensory areas in the human brain" Proceedings of the National Academy of Sciences of the USA $953295-3300$

Sanders A F J, Kappers A M L, 2006 "Bimanual curvature discrimination of hand-sized surfaces placed at different positions" Perception \& Psychophysics 68 1094-1106

Treutwein B, 1995 "Adaptive psychophysical procedures" Vision Research 35 2503-2522

Van Boven R W, Ingeholm J E, Beauchamp M S, Bikle P C, Ungerleider L G, 2005 "Tactile form and location processing in the human brain" Proceedings of the National Academy of Sciences of the USA $10212601-12605$

Wichmann F A, Hill N J, 2001a "The psychometric function: 1. Fitting, sampling, and goodness of fit" Perception \& Psychophysics $631293-1313$

Wichmann F A, Hill N J, 2001b "The psychometric function: II. Bootstrap-based confidence intervals and sampling" Perception \& Psychophysics $631314-1329$

Zhang M, Mariola E, Stilla R, Stoesz M, Mao H, Hu X, Sathian K, 2005 "Tactile discrimination of grating orientation: fMRI activation patterns" Human Brain Mapping 25 370-377 


\section{PERTEPTION}

VOLUME 372008

www.perceptionweb.com

Conditions of use. This article may be downloaded from the Perception website for personal research by members of subscribing organisations. Authors are entitled to distribute their own article (in printed form or by e-mail) to up to 50 people. This PDF may not be placed on any website (or other online distribution system) without permission of the publisher. 